\title{
Periodicity and Transport from Round-Off Errors
}

\author{
Franco Vivaldi
}

Dedicated the memory of Joseph Ford

\section{CONTENTS}

1. Introduction

2. Periodicity

3. Diffusion

4. A Stochastic Process

5. Discussion

Acknowledgements

Bibliography
We investigate the effects of round-off errors on quasi-periodic motions in a linear symplectic planar map. By discretizing coordinates uniformly we transform this map into a permutation of $\mathbb{Z}^{2}$, and study motions near infinity, which correspond to a fine discretization. We provide numerical evidence that all orbits are periodic and that the average order of the period grows linearly with the amplitude. The discretization induces fluctuations of the invariant of the continuum system. We investigate the associated transport process for time scales shorter than the period, and we provide numerical evidence that the limiting behaviour is a random walk where the step size is modulated by a quasi-periodic function. For this stochastic process we compute the transport coefficients explicitly, by constructing their generating function. These results afford a probabilistic description of motions on a classical invariant torus.

\section{INTRODUCTION}

In this paper we consider the effects of discretizing the phase space of a smooth area-preserving planar mapping that supports quasi-periodic motions, and we study the statistical properties of the resulting discrete representations of invariant tori.

Discretization of the phase space can be achieved in many ways, and the most natural ones amount to restricting the dynamics to discrete subsets of the phase space, most notably those containing periodic orbits. For instance, in the case of the Anosov diffeomorphisms of the torus - a class of strongly chaotic systems - the periodic orbits correspond to points with rational coordinates, which can be effectively represented as algebraic numbers in the field generated over the rationals by the eigenvalues of the mapping [Percival and Vivaldi 1987; Bartuccelli and Vivaldi 1989; Keating 
1991]. In the case of polynomial mappings over the complex field, the relevant discrete sets are towers of algebraic number fields containing the periodic and eventually periodic orbits [Vivaldi and Hatjispyros 1992; Bousch 1992; Morton and Patel 1994]. The thermodynamical formalism and the apparatus of dynamical zeta functions provide a link between the dynamics over these discrete sets and the statistics of their continuous counterpart (see [Ruelle 1978; Artuso et al. 1990; Artuso 1991] and references therein).

The discretization introduced by round-off is significantly more difficult to deal with. In this case the discrete and continuous systems are related only weakly, the discrete phase space does not have any useful algebraic structure, there is no framework for relating periodicity to the statistics of fluctuations, and the limit of fine discretization is plagued by singularities and slow convergence. (For a survey on this issue from an ergodic-theory angle, see [Blank 1989; 1994]. Some aspects of the relationship between exact and computed orbits are addressed by shadowing theory; see, for example, [Hammel et al. 1987; Blank 1991b].)

Computers make routine use of the floating-point representation of the reals, which consists of a finite subset of the rationals [Knuth 1981, Vol. 2, Ch. 4]. This set is not closed under any of the four arithmetical operations (even if we ignore overflow and underflow), so the dynamics does not restrict naturally to it. Moreover, the floating-point numbers cluster exponentially about zero, so that as a rule the floating-point dynamics is not a permutation, even when the map being represented is invertible.

Yet every symplectic map is a permutation, and any discrete model that is to be consistent with symplectic geometry must possess this property. Permutations can sometimes be constructed by discretizing coordinates uniformly, that is, by replacing the reals with the integers. In this case arithmetical errors may result only from division.

The uniform discretization of a symplectic map was first studied in [Rannou 1974]. This device was subsequently used in order to mimic quantum ef- fects [Chirikov et al. 1981], to achieve invertibility in a delicate numerical experiment [Karney 1983], to represent maps as cellular automata [Kaneko 1988], and to construct numerically reversible symplectic integrators [Scovel 1991]. A detailed numerical study of computer dynamics in the standard map was performed in [Earn and Tremaine 1992], where both reversible and floating-point discretizations were considered.

The statistical effects of round-off are sometimes likened to those of a small stochastic perturbation, and as such can be expected to generate a diffusive process, which interacts with the dynamics in a complicated manner.

However, this picture requires careful verification [Blank 1989; 1994]. The very fact that the computer arithmetic is well-defined implies that the round-off errors have null Kolmogorov complexity, that is, they have a nonrandom dependence on coordinates [Alexeev and Yakobson 1981; Ford 1983]. Thus, along any nonrandom (nonchaotic) orbit, the round-off perturbation cannot depend randomly on time, which hardly justifies the stochastic model. Of equal significance is the fact that round-off alone cannot induce exponential instability, since the error per iteration is bounded by the size of the discretization. Thus it is a matter of both theoretical and practical significance to ascertain what kind of statistical behaviour round-off perturbations can generate.

In this work we consider the simple setting of a uniform discretization of the phase space of a linear planar mapping that supports quasi-periodic motions. The continuous mapping is discretized in such a way as to become a permutation of $\mathbb{Z}^{2}$. In this model the size of the discretization is fixed, and the asymptotics of fine discretization correspond to motions near infinity. Because the continuous motions are regular and very well understood, the effect of round-off will be isolated quite clearly.

In Section 2 we introduce our model (2.1) and investigate the asymptotics of its periodic behaviour. We show that the period of the orbits must diverge at infinity, and derive a lower bound for its growth 
rate as a function of the amplitude (Theorem 2.1). We then provide numerical evidence that the average order of the period grows linearly with the amplitude, that is, with the inverse of the discretization length (a comparison with the dissipative case is instructive; see [Beck and Roepstorff 1987]).

In Sections 3 and 4 we deal with the transport problem. We analyze the dynamical evolution of the unperturbed (continuum) invariant, and show that its irregular behaviour originates from the interaction between two frequencies whose ratio becomes singular in the limit. We then investigate the associated transport process for time scales shorter than the period, and provide evidence that its asymptotic behaviour is that of a random walk whose step size is modulated by a quasi-periodic function. For this stochastic process we compute the transport coefficients by constructing their generating function (Theorem 4.1), and we conjecture that this function is the correct one for the roundoff problem as well.

Section 5 contains concluding remarks.

The results presented here suggest a probabilistic characterization of motions on a classical invariant torus. All reported computations refer to the golden mean rotation number, but we have occasionally tested other diophantine cases. We make no claim for frequencies that are well approximated by rationals, such as Liouville numbers.

We remark that the choice of the model (2.1) was dictated by mathematical considerations, not by a desire to simulate round-off errors in actual numerical experiments. For instance, we insist that the discrete mapping be a permutation, which is seldom the case in practice. For this reason, the question of the applicability of our results to more general situations should be considered with care.

\section{PERIODICITY}

We consider the following mapping $\Phi: \mathbb{Z}^{2} \mapsto \mathbb{Z}^{2}$ :

$$
\Phi(x, y)=(\lfloor\lambda x\rfloor-y, x),
$$

where $\lfloor x\rfloor$ is the largest integer smaller or equal to $x$ and $\lambda$ is a real parameter. This mapping is a permutation of $\mathbb{Z}^{2}$, as easily verified. Defining $\Psi: \mathbb{R}^{2} \mapsto \mathbb{R}^{2}$ by

$$
\Psi(x, y)=(\lambda x-y, x)
$$

and setting $\varepsilon(x)=\lambda x-\lfloor\lambda x\rfloor$, we may view $\Phi$ as a discrete approximation of $\Psi$, that is, $\Phi=\Psi-\varepsilon$. This interpretation becomes meaningful at large amplitudes, since $\varepsilon$ remains bounded: $0 \leq \varepsilon<$ 1. This asymptotic regime corresponds to a fine discretization.

The dependence of $\varepsilon$ on $x$ is periodic if $\lambda$ is rational, and quasi-periodic otherwise. In the former case $\varepsilon(\mathbb{Z})$ is a finite subset of rationals, uniformly spaced in the unit interval. In the latter, $\varepsilon(\mathbb{Z})$ consists of the number zero and a set of irrationals dense in the unit interval.

The mapping $\Psi$ admits the invariant quadratic form

$$
Q(x, y)=x^{2}-\lambda x y+y^{2},
$$

whose level sets are symmetrical with respect to the axes $x= \pm y$. We are interested in bounded quasi-periodic motions, corresponding to $|\lambda|<2$ and an irrational rotation number; the rotation number $\nu$ is related to $\lambda$ by $\lambda=2 \cos (2 \pi \nu)$. All orbits of the continuous system are dense on ellipses, and the stability problem for its discrete counterpart arises naturally. Combinatorially speaking, stability amounts to nonexistence of cycles of infinite length, which would correspond to orbits escaping to infinity in both time directions.

A few values of $\lambda$ lead to trivial dynamics. For $\lambda=1,0$ and -1 , we have $\varepsilon(x)=0$ for all $x$, the mapping $\Phi$ is a restriction of $\Psi$ to $\mathbb{Z}^{2}$, and all orbits are periodic with minimal period six, four and three, respectively. From cyclotomy one knows that for $|\lambda|<2$ these are the only cases in which both $\nu$ and $\lambda$ are rational (see [Marcus 1977, Ch. 2], for example), and for all other rational rotation numbers, $\lambda$ is irrational and algebraic.

The set of $\nu$ that corresponds to rational (noninteger) $\lambda$ is more difficult to characterize (it is 
tempting to conjecture that any such $\nu$ must be transcendental, although this issue seems difficult to settle). Letting $\lambda_{n}=2 \cos (2 \pi n \nu)$, for $n=$ $1,2, \ldots$, one sees that $\lambda_{1}=\lambda$ and $\lambda_{n}=2 C_{n}\left(\frac{1}{2} \lambda\right)$, where $C_{n}(x)$ is the $n$-th Chebyshev polynomial of the first kind. Since the latter has integral coefficients, all numbers $\lambda_{n}$ belong to the same field as $\lambda$.

If the rotation number is irrational, an invariant torus is typically represented by a cloud of points irregularly arranged along an unperturbed invariant set: see Figure 1 and [Earn and Tremaine 1992, Fig. 7].

In this case the period must diverge at infinity, as established by the following result:

Theorem 2.1. Let $T(x, y)$ be the (possibly infinite) period of the orbit of $\Phi$ through the point $(x, y)$, and let

$\tau(r)=\min \left\{T(x, y):(x, y) \in \mathbb{Z}^{2}\right.$ and $\left.Q(x, y) \geq r^{2}\right\}$.

Then, if $\nu$ is irrational, $\lim _{r \rightarrow \infty} \tau(r)=\infty$. Moreover, for almost all values of $\nu$ and any $\delta>0$, there exists a positive constant $c$ such that

$$
\tau(r)>c r^{1 /(2+\delta)}
$$

Proof. Let $\nu$ be irrational, and let $p_{n} / q_{n}$ be a convergent of its continued fraction expansion. The following inequality holds [Khinchin 1964, Theorem 13]:

$$
\left|\frac{p_{n}}{q_{n}}-\nu\right|>\frac{1}{q_{n}\left(q_{n}+q_{n+1}\right)}
$$

for $n=0,1, \ldots$ Let $w=(u, v) \in \mathbb{Z}^{2}$. Now convergents of continued fractions are the best rational approximants of the second kind [Khinchin 1964, theorem 17]. Therefore, if $J$ is the Jordan form of $\Psi$-that is, a rotation by $2 \pi \nu$ - the point $J^{q_{n}} w$ is the closest to $w$ among the first $q_{n}$ points in the orbit. Letting $r^{2}=u^{2}+v^{2}$, the estimate (2.4) yields

$$
\left\|J^{t} w-w\right\|>\frac{4 r}{q_{n}+q_{n+1}}
$$

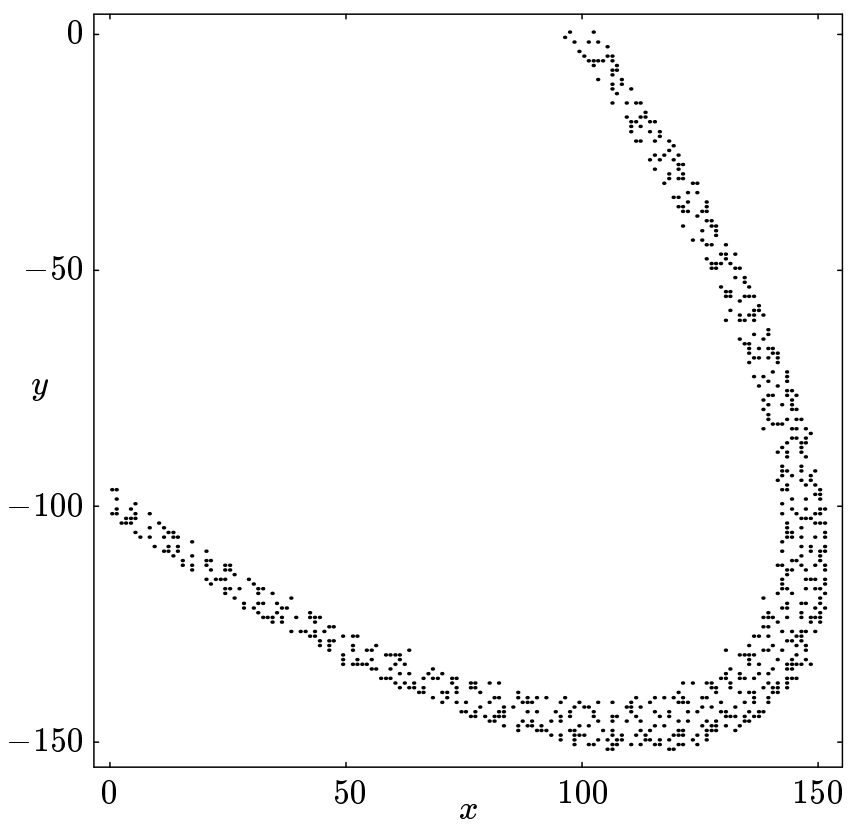

FIGURE 1. A discrete representation of an invariant torus of golden mean rotation number, by a periodic orbit of period 1927.

for $n=0,1, \ldots$ and $t=1, \ldots, q_{n}$, where $\|\cdot\|$ is the euclidean metric on the plane.

Let $M$ be a linear conjugacy between $\Psi$ and its Jordan form, so that $\Psi=M J M^{-1}$, and let $M w=$ $z=(x, y)$. We have

$$
\begin{aligned}
\left\|J^{t} w-w\right\| & =\left\|M^{-1}\left(M J^{t} w-M w\right)\right\| \\
& =\left\|M^{-1}\left(\Psi^{t} z-z\right)\right\| \\
& \leq \kappa\left\|\Psi^{t} z-z\right\|
\end{aligned}
$$

where $\kappa$ is the norm of $M^{-1}$. Without loss of generality we may assume that $M$ is chosen so as to preserve the value of the corresponding form, that is, $u^{2}+v^{2}=Q(x, y)=r^{2}$. Then, combining (2.5) and (2.6), we obtain

$$
\left\|\Psi^{t} z-z\right\|>\frac{4 r}{\kappa} \frac{1}{q_{n}+q_{n+1}}
$$

for $n=0,1, \ldots$ and $t=1, \ldots, q_{n}$. Let $\mu_{t}$ be the norm of $\Psi^{t}$, and let $\mu=\sup _{t \in \mathbb{Z}} \mu_{t}$. Then $\mu<\infty$ 
because the motion is bounded, and the following estimate holds:

$$
\left\|\Phi^{t} z-\Psi^{t} z\right\|<\mu t \quad \text { for } t \geq 1 .
$$

Indeed this inequality holds for $t=1$, because

$$
\|\Phi z-\Psi z\|<1
$$

for all $z \in \mathbb{Z}^{2}$, and $\mu \geq 1$ since $\Psi$ is area-preserving. Assuming (2.8) to be true for some $t \geq 1$, we have

$$
\begin{aligned}
\left\|\Phi^{t+1} z-\Psi^{t+1} z\right\| & \\
& \leq\left\|\Phi^{t+1} z-\Psi^{t} \Phi z\right\|+\left\|\Psi^{t+1} z-\Psi^{t} \Phi z\right\| \\
& =\left\|\Phi^{t} \Phi z-\Psi^{t} \Phi z\right\|+\left\|\Psi^{t}(\Psi z-\Phi z)\right\| \\
& <t \mu+\mu\|\Phi z-\Psi z\|<(t+1) \mu,
\end{aligned}
$$

which completes the induction. Using the triangular inequality we conclude from (2.7) and (2.8) that

$$
\left\|\Phi^{t} z-z\right\|>\frac{4 r}{\kappa} \frac{1}{q_{n}+q_{n+1}}-\mu q_{n}
$$

for $n=0,1, \ldots$ and $t=1, \ldots, q_{n}$. The right-hand side of this inequality is positive (implying that $\left.T(x, y)>q_{n}\right)$ for all sufficiently large $r$-more precisely, for

$$
r>\frac{1}{4} \kappa \mu\left(q_{n}^{2}+q_{n} q_{n+1}\right) .
$$

This shows that $\tau(r)>q_{n}$ for such values of $r$. The first assertion of the theorem now follows from the fact that $q_{n} \rightarrow \infty$.

To prove the estimate (2.3) we note that the convergence of the integral

$$
\int_{a}^{\infty} \frac{d q}{q^{1+\delta}}
$$

(where $a>0$ and $\delta>0$ ) implies that, for almost all rotation numbers $\nu$, there exists a constant $c^{\prime}$ such that the inequality

$$
\left|\frac{p}{q}-\nu\right|>\frac{c^{\prime}}{q^{2+\delta}}
$$

is satisfied for all positive integers $p$ and $q$ [Khinchin 1964, Theorem 32]. Replacing (2.4) with this inequality and repeating the above argument, we conclude that $\tau>q_{n}$ for

$$
r>\frac{\kappa \mu}{4 c^{\prime}} q_{n}^{2+\delta}
$$

from which the desired result follows.

The bound (2.3) is too weak for an effective computation of the function $\tau$. For this reason, in place of $\tau$ we consider the simpler function $f(r)=T(r, 0)$, which measures $T$ on a single representative point of the level set $Q(x, y)=r^{2}$, for integer $r$. Clearly, $f(r) \geq \tau(r)$. This simplification is not as crude as it may seem: we have found numerically, by sampling a few rotation numbers, that some two-thirds of the orbits have a representative point on the $x$ axis (which is not a symmetry axis).

A log-log plot of $f(r)$ for the golden mean rotation number $\nu=\frac{1}{2}(1+\sqrt{5})$ is displayed in Figure 2 . The large fluctuations are not unexpected, given the arithmetical nature of $f$. The lower envelope represents a lower bound of the form $c r^{2 / 3}$, which suggest that the bound (2.3) can be improved. The upper bound is somewhat greater than linear (the

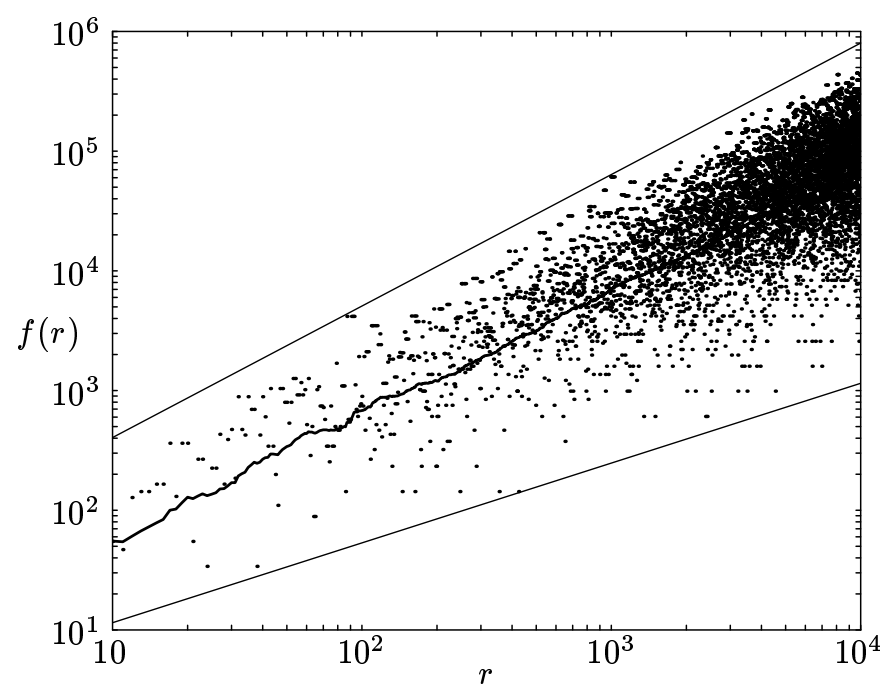

FIGURE 2. The period $f(r)$ of the orbits through the points $(x, y)=(r, 0)$. The solid curve represents the average order of $f$. The lower and upper envelopes have slope $\frac{2}{3}$ and $\frac{11}{10}$. 
exponent is equal to $\left.\frac{11}{10}\right)$. The thick solid curve represents the average order $F$ of $f$, given by

$$
F(r)=\frac{1}{r} \sum_{k \leq r} f(k),
$$

which is introduced to suppress fluctuations.

Figure 3 plots $F(r) / r$ for the same rotation number. Comparison between domain and range shows that, asymptotically, $F$ is dominated by a linear term, but the persisting fluctuations leave doubts about the existence of a limit.

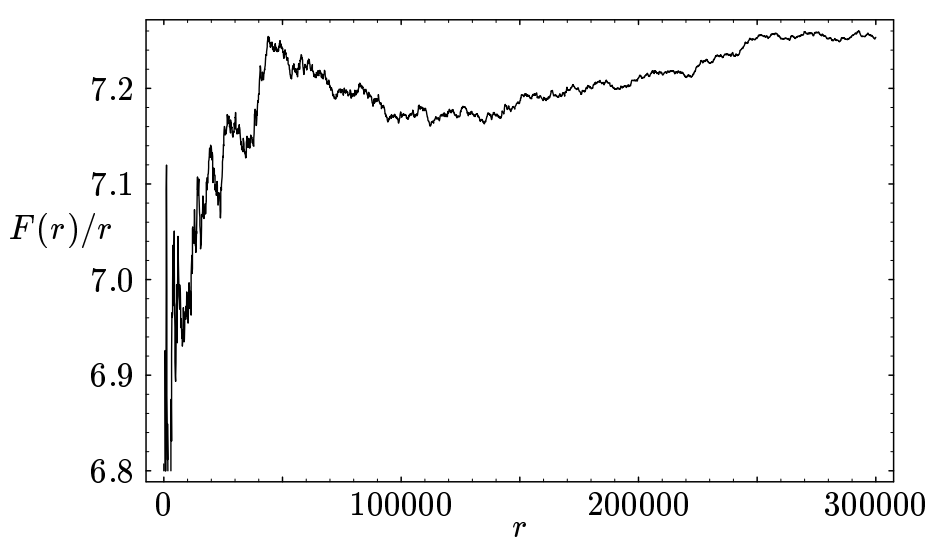

FIGURE 3. The function $F(r) / r$ for the golden mean, where $F$ is the average order of $f$, given by $(2.9)$.

\section{DIFFUSION}

We consider variations of the $\Psi$-invariant form $Q$ defined in (2.2). Let $\left(x_{t+1}, y_{t+1}\right)=\Phi\left(x_{t}, y_{t}\right)$. We have

$$
Q\left(x_{t+1}, y_{t+1}\right)=Q\left(x_{t}, y_{t}\right)+\Delta Q\left(x_{t}, y_{t}\right),
$$

where

$$
\begin{aligned}
\Delta Q\left(x_{t}, y_{t}\right) & =\left(2 y_{t}-\lambda x_{t}\right) \varepsilon\left(x_{t}\right)+\varepsilon\left(x_{t}\right)^{2} \\
& =\left(y_{t}-x_{t+1}\right) \varepsilon\left(x_{t}\right) .
\end{aligned}
$$

In place of $\Delta Q$ we consider the normalized function $\Theta(x, y)=\varepsilon(x) \eta(x, y)$, where

$$
\eta(x, y)=\frac{2 y-\lambda x}{2 \sqrt{Q(x, y)}} .
$$

We have $|\eta(x, y)| \leq 1$ because

$$
|2 y-\lambda x| \leq 2 \sqrt{Q(x, y)}
$$

and, since $|\varepsilon(x)|<1$, the function $\Theta$ is normalized: $|\Theta(x, y)| \leq 1$.

For the continuum map $\Psi$, the function $\eta=\eta(t)$ can be computed explicitly, giving

$$
\eta(t)=\cos (2 \pi \nu t+\theta)
$$

where $\theta$ is a constant depending on $\lambda$ as well as on the initial conditions. Thus, if $\nu$ is irrational, $\eta$ is quasi-periodic, with invariant density

$$
\rho_{\eta}(z)=\frac{1}{\pi \sqrt{1-z^{2}}}
$$

for $z=\eta(x, y),|z|<1$. This distribution, which is independent of $\lambda$, is well approximated by that computed on a sufficiently long discrete orbit (see Figure 4, top left).

Our $\varepsilon$ is the source of fluctuations. When $\lambda$ is irrational, the values of $\varepsilon$ are distributed uniformly in the unit interval, although the fluctuations are larger that those of $\eta$ (Figure 4, top right). Assuming $\varepsilon$ and $\eta$ to be independent, one finds that $\Theta$ has density

$$
\rho_{\Theta}(z)=\frac{1}{\pi} \ln \left(\frac{1+\sqrt{1-z^{2}}}{|z|}\right)
$$

for $z=\Theta(x, y),|z|<1$, which is compared with the density computed on a discrete orbit in Figure 4 , bottom, showing a good agreement. This result verifies numerically the independence of $\varepsilon$ and $\eta$. (Adapting the above consideration to the case of rational $\lambda$ is not difficult, but we shall not do it here.)

The normalized variation $\chi(t)$ of $Q$ after $t$ iterations is defined by

$$
\begin{aligned}
\chi\left(t, x_{0}, y_{0}\right) & =\sum_{k=0}^{t-1} \Theta\left(x_{k}, y_{k}\right) \\
& \simeq \frac{Q\left(x_{t}, y_{t}\right)-Q\left(x_{0}, y_{0}\right)}{2 \sqrt{Q\left(x_{0}, y_{0}\right)}} .
\end{aligned}
$$



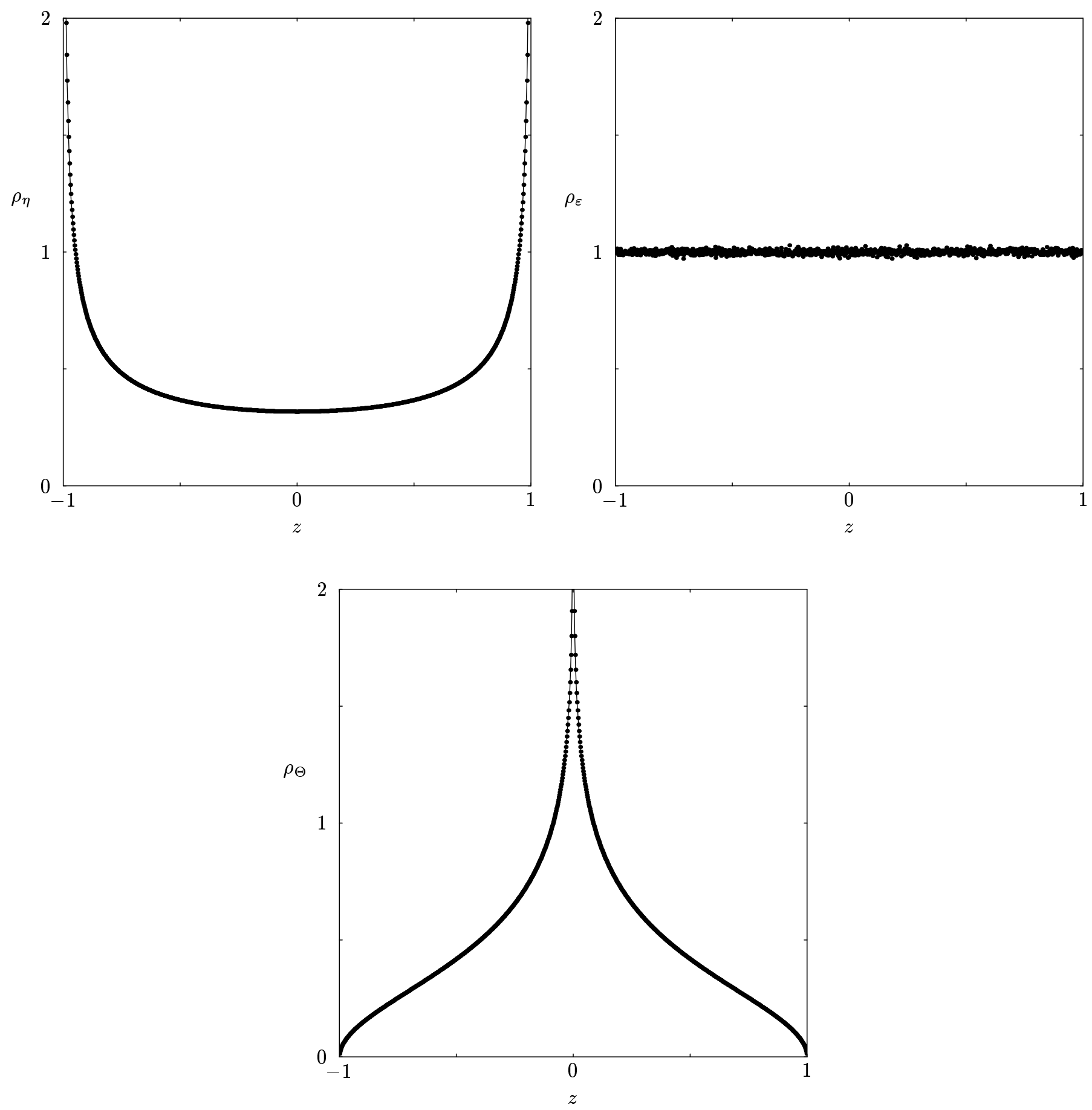

FIGURE 4. Probability distributions computed along an orbit of period 25805783. Top left: distribution for the variable $\eta$. The solid curve represents the estimate (3.1). Top right: distribution for $\varepsilon$. Bottom: Distribution for $\Theta$. The solid curve represents the estimate $(3.2)$. 
A typical evolution of $\chi$ over a time interval shorter than the period is displayed in Figure 5, which suggests that this process possesses nontrivial statistical properties. Because the period of the orbits diverges at infinity, it is possible to investigate the long-time asymptotics of (3.3) on suitable sequences of periodic orbits.

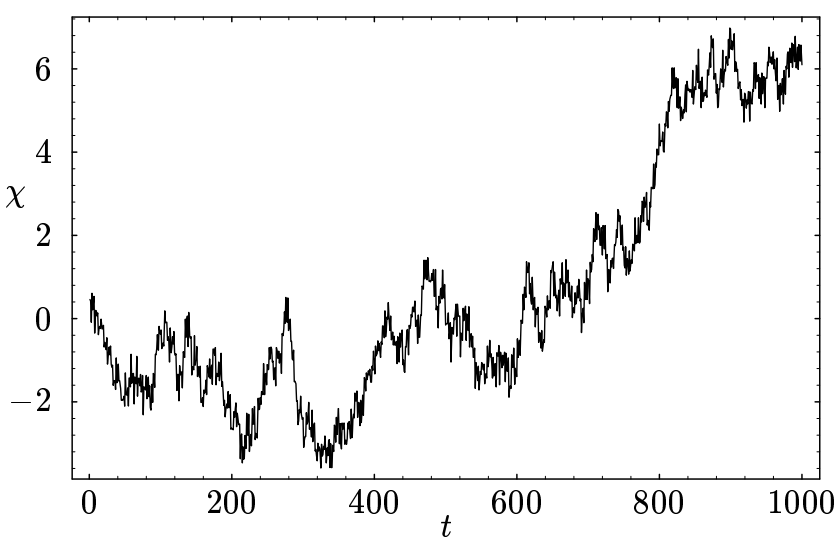

FIGURE 5. A typical evolution of $\chi(t)$, computed along an orbit of period 7553 .

We are interested in the long-time behaviour of the cumulants $\kappa_{k}(t)$ of the distribution of $\chi(t)$, computed according to some average $\langle\cdot\rangle$ (to be defined below) with respect to the initial point $\left(x_{0}, y_{0}\right)$. First we write the (as yet formal) exponential generating function for the cumulants [David and Barton 1962, Ch. 3]:

$$
K_{t}(z)=\ln \langle\exp (z \cdot \chi(t))\rangle=\sum_{k=1}^{\infty} \kappa_{k}(t) \frac{z^{k}}{k !}
$$

for $t=1,2, \ldots$ We then define [Artuso 1991; Wang and $\mathrm{Hu}$ 1993]

$$
\begin{aligned}
P(z) & =\lim _{t \rightarrow \infty} \frac{1}{t} K_{t}(z)=\lim _{t \rightarrow \infty} \sum_{k=1}^{\infty} \frac{\kappa_{k}(t)}{t} \frac{z^{k}}{k !} \\
& =\lim _{t \rightarrow \infty} \sum_{k=1}^{\infty} p_{k}(t) \frac{z^{k}}{k !},
\end{aligned}
$$

where $p_{k}(t)=\kappa_{k}(t) / t$. If $K_{t}$ is analytic at zero for all $t$, and if the limit (3.4) exists for all $k$, then $P$ is analytic at zero, and it becomes the exponential generating function of the transport coefficients $p_{k}$

$$
P(z)=\sum_{k=1}^{\infty} p_{k} \frac{z^{k}}{k !} .
$$

If the limit (3.4) does not exist for some $k$, one speaks of anomalous transport (see, for example, [Wang and $\mathrm{Hu}$ 1993; Artuso et al. 1992] and references therein).

We are interested in the behaviour of $p_{k}(t)$ for small values of $k$. Letting $\mu_{k}=\left\langle\chi(t)^{k}\right\rangle$ be the $k$ th moment of $\chi$, standard theory gives [David and Barton 1962, p. 43]

$$
\begin{aligned}
& \kappa_{1}=\mu_{1}, \\
& \kappa_{2}=\mu_{2}-\mu_{1}^{2}, \\
& \kappa_{3}=\mu_{3}-3 \mu_{1} \mu_{2}+\mu_{1}^{3}, \\
& \kappa_{4}=\mu_{4}-3 \mu_{2}^{2}-4 \mu_{1} \mu_{3}+12 \mu_{2} \mu_{1}^{2}-6 \mu_{1}^{4}, \\
& \kappa_{5}=\mu_{5}-5 \mu_{4} \mu_{1}-10 \mu_{3} \mu_{2}+20 \mu_{3} \mu_{1}^{2}+30 \mu_{2}^{2} \mu_{1}-60 \mu_{2} \mu_{1}^{3} \\
& \quad+24 \mu_{1}^{5}, \\
& \kappa_{6}=\mu_{6}-6 \mu_{5} \mu_{1}-15 \mu_{4} \mu_{2}+30 \mu_{4} \mu_{1}^{2}-10 \mu_{3}^{2}+120 \mu_{3} \mu_{2} \mu_{1} \\
& \quad-120 \mu_{3} \mu_{1}^{3}+30 \mu_{2}^{3}-270 \mu_{2}^{2} \mu_{1}^{2}+360 \mu_{2} \mu_{1}^{4}-120 \mu_{1}^{6} .
\end{aligned}
$$

We have computed the functions $p_{k}(t)$ for the process (3.3), for $1 \leq k \leq 6$ and $t \leq 200$. For each value of $t$, the phase averaging was done over the points of a single orbit of period 1128946 (Figure 6, top) and 116803411 (bottom). (A more rigorous averaging procedure will be discussed below.) Although all functions $p_{k}(t)$ are necessarily periodic, with period equal to that of the orbit, we are only interested in the very early part of the period, which corresponds to relaxation to the regime of infinite period.

For the first three odd values of $k$, the function $p_{k}$ was found to vanish identically within numerical accuracy, as expected from the symmetry of the $\Theta$-distribution. In both panels of Figure 6 we have plotted the first three even transport functions $p_{2}(t), p_{4}(t)$ and $p_{6}(t)$. The dotted lines represent their conjectured limiting values

$$
p_{2}=\frac{1}{24}, \quad p_{4}=-\frac{1}{320}, \quad p_{6}=\frac{5}{4032}
$$


(see next section); $p_{2}$ is the diffusion coefficient and $p_{4}$ is the Burnett coefficient. For fixed $t$, the convergence of $p_{2 k}(t)$, in terms of the size of the averaging sample, becomes quickly troublesome as $k$ increases. Within the relatively limited statistics of Figure 6 (top), only the diffusion coefficient $(k=2)$ appears to relax to some limiting value, while the other coefficients fluctuate wildly. The improved statistics of Figure 6 (bottom) confirms
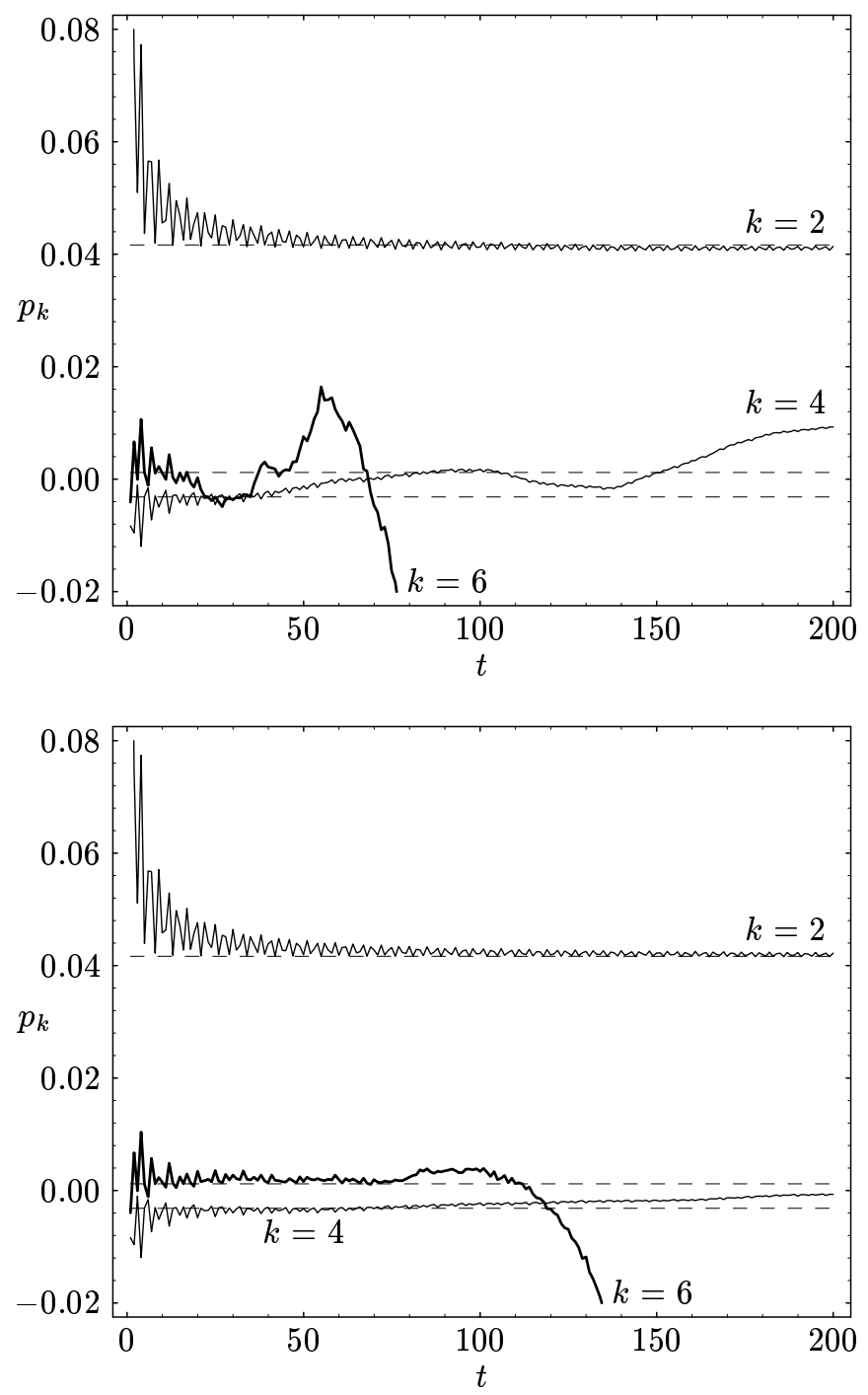

FIGURE 6. The transport functions $p_{k}(t)$, for $k=2$, 4 and 6 , computed by averaging over all points of a periodic orbit of period 1128946 (top) and 116803411 (bottom). the convergence of $p_{2}$. The behaviour of the other coefficients is consistent with convergence to the limits (3.6), although at this stage the evidence is rather weak. In Section 4, we shall strengthen this argument by analyzing the asymptotics of a stochastic process closely related to (3.3), which is amenable to analytical investigation.

From a theoretical angle, the averaging over a single long orbit adopted above is unsatisfactory. A firmer procedure could consist in first averaging over all lattice points belonging to sufficiently long periodic orbits, and then letting the period go to infinity. More precisely, let $W=W(t)$ be the set of lattice points $z=(x, y) \in \mathbb{Z}^{2}$ for which $T(z)>t$, and let $V(r, t)$ be the intersection of $W(t)$ with the solid ellipses $Q(z) \leq r^{2}$. For a lattice function $g$ we define the average

$$
\langle g\rangle_{t}=\lim _{r \rightarrow \infty} \frac{1}{|V(r, t)|} \sum_{z \in V(r, t)} g(z)
$$

assuming that the limit exists. Because the complement of $W$ in $\mathbb{Z}^{2}$ is finite (from Theorem 2.1), the set $W(t)$ will include the exterior of a sufficiently large ellipses, whence $|V(r, t)| \sim r^{2}$. Finally

$$
\langle g\rangle=\lim _{t \rightarrow \infty}\langle g\rangle_{t} .
$$

We conjecture that for all positive integers $k$ and $s$, the functions $p_{k}(s)$ converge with respect to this average.

\section{A STOCHASTIC PROCESS}

In order to strengthen the case for a probabilistic interpretation of the round-off fluctuations, and to support the interpretation of the results presented in the previous section, we compare the deterministic process (3.3) with the stochastic process

$$
\bar{\chi}(t)=\sum_{s=1}^{t} \bar{\varepsilon}_{s} \sin (2 \pi \nu s),
$$

where the $\bar{\varepsilon}_{s}$ are random variables uniformly distributed in the unit interval. The key assumptions behind the introduction of (4.1) as a model for 
(3.3) are the randomness of $\varepsilon$ and its independence from $\eta$.

The long-time statistical behaviour of the process (4.1) is described by the following result:

Theorem 4.1. Let $\bar{\chi}(t)$ be given by (4.1) and let $\nu$ be irrational. Then, for $|z|<2 \pi$, we have

$$
\begin{aligned}
\bar{P}(z) & =\lim _{t \rightarrow \infty} \frac{1}{t} \ln \langle\exp (z \cdot \bar{\chi}(t))\rangle \\
& =\sum_{n=1}^{\infty} \frac{B_{2 n}}{2^{2 n+1} n(n !)^{2}} z^{2 n},
\end{aligned}
$$

where $B_{k}$ is the $k$-th Bernoulli number.

A comparison of the right-hand side of this equation with (3.5) yields at once the transport coefficients

$$
p_{2 n}=\frac{(2 n-1) ! B_{2 n}}{2^{2 n}(n !)^{2}}
$$

for $n=1,2, \ldots$, which is the formula used to obtain (3.6).

Before proving the theorem, we estimate the errors in the numerical evaluation of $p_{k}(t)$ (for fixed $t)$ that result from the finiteness of the averaging sample. The data displayed in Figure 7 were obtained by averaging over 100 million values of the

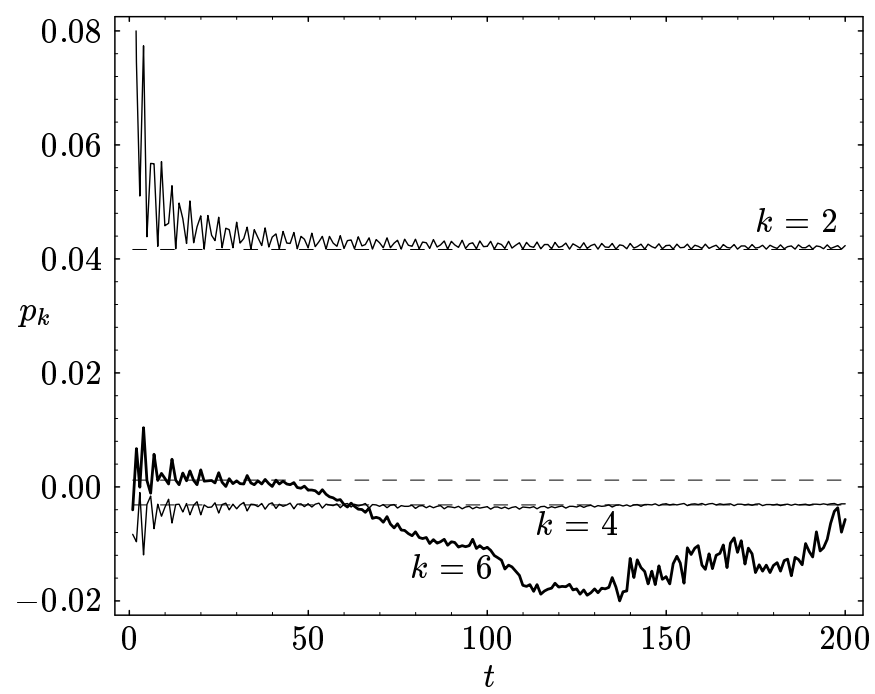

FIGURE 7. The transport functions of the stochastic process (4.1), computed by averaging over 100 million data. random variable $\bar{\varepsilon}(k)$ produced by a random number generator, so as to obtain a statistics similar to that of Figure 6 (bottom). The difference between the behaviour of (3.3) and (4.1) was found to be comparable with the fluctuations observed by changing random sequence. On this basis, we conclude that these results are consistent with the hypothesis that the two processes share not only the same asymptotics, but also all functions $p_{k}(t)$. Proof of Theorem 4.1. Let $\bar{\eta}_{s}=\sin (2 \pi \nu s)$. Then $\bar{\Theta}_{s}=\bar{\varepsilon}_{s} \bar{\eta}_{s}$ is a random variable, with density

$$
\rho_{s}(x)= \begin{cases}\left|\bar{\eta}_{s}\right|^{-1} & x \in\left[0, \bar{\eta}_{s}\right] \\ 0 & \text { otherwise }\end{cases}
$$

Because the variables $\Theta_{s}$ are independent, the cumulants of $\chi(t)$ are the sum of the cumulants of the $\Theta$, that is,

$$
\bar{P}(z)=\lim _{t \rightarrow \infty} \frac{1}{t} \sum_{s=1}^{t} \ln \left\langle\exp \left(z \cdot \bar{\Theta}_{s}\right)\right\rangle .
$$

We have

$$
\left\langle\exp \left(z \cdot \bar{\Theta}_{s}\right)\right\rangle=\sum_{k=0}^{\infty}\left\langle\bar{\Theta}_{s}^{k}\right\rangle \frac{z^{k}}{k !} .
$$

From (4.2) we compute

$$
\left\langle\bar{\Theta}_{s}^{k}\right\rangle=\frac{\eta_{s}^{k}}{k+1}
$$

which, combined with (4.4), yields

$$
\left\langle\exp \left(z \cdot \bar{\Theta}_{s}\right)\right\rangle=\frac{1}{z \bar{\eta}_{s}}\left(e^{z \bar{\eta}_{s}}-1\right) .
$$

Thus (4.3) becomes

$$
\bar{P}(z)=\lim _{t \rightarrow \infty} \frac{1}{t} \sum_{s=1}^{t} \ln \frac{e^{z \bar{\eta}_{s}}-1}{z \bar{\eta}_{s}} .
$$

For irrational $\nu$, the sequence $s \mapsto 2 \pi \nu s \bmod 2 \pi$ is uniformly distributed in the interval $[0,2 \pi]$. Because the logarithmic function in this last expres- 
sion is continuous in that interval, we obtain, from the ergodic theorem,

$$
\bar{P}(z)=\frac{1}{2 \pi} \int_{-\pi}^{\pi} \ln \frac{e^{z \sin (x)}-1}{z \sin (x)} d x .
$$

Let $y=\frac{1}{2} z \sin (x)$. We rewrite the integrand above as

$\ln \frac{e^{2 y}-1}{2 y}=y+\ln \frac{\sinh y}{y}=y+\sum_{k=1}^{\infty} \ln \left(1+\left(\frac{y}{\pi k}\right)^{2}\right)$.

The first term is odd in $x$ and integrates to zero, and we get

$$
\begin{aligned}
\bar{P}(z) & =\frac{1}{\pi} \int_{-\pi / 2}^{\pi / 2} \sum_{k=1}^{\infty} \ln \left(1+\left(\frac{y}{\pi k}\right)^{2}\right) d x \\
& =\frac{2}{\pi} \sum_{k=1}^{\infty} \int_{0}^{\pi / 2} \ln \left(1+\left(\frac{z}{2 \pi k}\right)^{2} \sin ^{2}(x)\right) d x .
\end{aligned}
$$

Integrating we obtain (see [Gradshteyn and Ryzhik 1980, Eq. 4.399], for example):

$$
\bar{P}(z)=2 \sum_{k=1}^{\infty} \ln \frac{1+\sqrt{1+(z / 2 \pi k)^{2}}}{2} .
$$

Next we expand the logarithmic function [Gradshteyn and Ryzhik 1980, Eq. 1.515] to obtain, for $|z|<2 \pi$,

$$
\begin{aligned}
\bar{P}(z) & =\sum_{k=1}^{\infty} \sum_{n=1}^{\infty}(-1)^{n+1} \frac{2(2 n-1) !}{2^{2 n}(n !)^{2}}\left(\frac{1}{2 \pi k}\right)^{2 n} z^{2 n} \\
& =\sum_{n=1}^{\infty}(-1)^{n+1} \frac{2(2 n-1) !}{2^{2 n}(n !)^{2}(2 \pi)^{2 n}} \zeta(2 n) z^{2 n},
\end{aligned}
$$

where $\zeta$ is the Riemann zeta function. For any positive integer $n$ we have [Apostol 1984, Theorem 12.17]

$$
\zeta(2 n)=(-1)^{n+1} \frac{(2 \pi)^{2 n}}{2(2 n) !} B_{2 n},
$$

where the $B_{k}$ are the Bernoulli numbers. Combining this with the value of $\bar{P}(z)$ yields the desired formula.

\section{DISCUSSION}

In this work we have explored the dynamical and statistical effects of round-off errors, in a model problem where the coordinates of a regular dynamical system - linear quasi-periodic motions on tori-are discretized uniformly.

As it is frequently the case in discrete dynamics, obstructions to predictability originate from computational difficulties of the time complexity type (programs take too long to run). This is in sharp contrast with the case of continuum systems, where randomness and unpredictability are rooted in the positive algorithmic complexity of the orbits (programs are too large) [Alexeev and Yakobson 1981; Ford 1983].

The problem of predicting the period of an orbit (which was found to depend irregularly on coordinates) serves as an illustration. Specifying a lattice point near the invariant curve $Q(x, y)=r^{2}$ requires $O\left(\log _{2}(r)\right)$ bits of information, with which we can generate an orbit whose period grows faster than logarithmically. Thus the algorithmic complexity of any orbit is zero.

On the other hand, the mechanism that causes an orbit to become periodic has a strong probabilistic flavour. Even though the period-particularly when small - tends to be the denominator of a good rational approximant of the rotation number (often a convergent of its continued fractions), the sheer size of the fluctuations raises the question of whether or not a polynomial-time algorithm for computing the period exists. Without that, the only computational strategy would be essentially "wait and see", which would make this problem intractable. For instance, the computation of Figure 3 , based on direct iteration, required some 600 hours of CPU time on a Sun Sparcstation 2.

A natural question concerns the existence of a "continuum limit", which can be defined, for instance, in terms of convergence of discrete invariant measures to the continuum ones [Blank 1989]. This question is closely related to the growth rate of the period of the orbits. Indeed in order to en- 
sure that, asymptotically, the discrete torus has zero relative "thickness", one must establish that the period of the orbits grows slower than $r^{2}$. Our results lead us to conjecture that such bound exists for (almost all) irrational rotation numbers. However, we have also found strong indications that for some rational rotation numbers the continuum limit - in the sense indicated above - may not exist. (The boundedness of all orbits of a uniform discretization of an irrational symplectic rotation was first conjectured in [Blank 1991a].)

The main open question on the transport problem is to decide whether or not the generating function (3.4) is actually given by the function $\bar{P}$ of Theorem 4.1. We believe this to be the case, although the evidence presented here is not conclusive. In particular, one cannot rule out nonanalytic behaviour for $P$, that is, anomalous transport.

These problems are currently under investigation.

\section{ACKNOWLEDGEMENTS}

I am grateful to Michael Blank for bringing several references to my attention, and to Ya. G. Sinai for a useful clarification concerning the material presented in Section 4.

\section{REFERENCES}

[Alexeev and Yakobson 1981] V. M. Alexeev and M. V. Yakobson, "Symbolic dynamics and hyperbolic dynamical systems", Phys. Reports 75 (1981), 287325 .

[Apostol 1984] T. A. Apostol, Introduction to analytic number theory, Springer, New York, 1984.

[Artuso 1991] R. Artuso, "Diffusive dynamics and periodic orbits of dynamical systems", Phys. Lett. A160 (1991), 528-530.

[Artuso et al. 1990] R. Artuso, E. Aurell, and P. Cvitanović, "Recycling strange sets: I. Cycle expansions", Nonlinearity 3 (1990), 325-359.

[Artuso et al. 1992] R. Artuso, G. Casati and D. L. Shepelyanski, "Fractal spectrum and anomalous diffusion in the kicked Harper model", Phys. Rev. Lett. E68 (1992), 3826-3829.

[Bartuccelli and Vivaldi 1989] M. Bartuccelli and F. Vivaldi, "Ideal orbits of toral automorphisms", Physica D39 (1989), 194-204.

[Beck and Roepstorff 1987] C. Beck and G. Roepstorff, "Effects of phase space discretization on the longtime behavior of dynamical systems", Physica D25 (1987), 173-180.

[Blank 1989] M. Blank, "Small perturbations of chaotic dynamical systems", Russian Math. Surveys $\mathbf{4 4}$ (1989), 1-33. Reprinted in Dynamical systems and statistical mechanics (edited by Ya. G. Sinai), Adv. in Sov. Math., 3, Amer. Math. Soc., Providence, RI, 1991.

[Blank 1991a] M. Blank, "Marginal singularities, almost invariant sets, and small perturbations", Chaos 1 (1991), 347-356.

[Blank 1991b] M. Blank, "Phase space discretization in chaotic dynamical systems", pp. 1-13 in Dynamical systems and statistical mechanics (edited by Ya. G. Sinai), Adv. in Sov. Math. 3, Amer. Math. Soc., Providence, RI, 1991.

[Blank 1994] M. Blank, Pathologies generated by round-off in dynamical systems, Physica D78 (1994), 93-114.

[Bousch 1992] T. Bousch, "Sur quelques problèmes de la dynamique holomorphe", Ph.D. thesis, Université de Paris-Sud, Centre d'Orsay, 1992.

[Chirikov et al. 1981] Chirikov, F. M. Izrailev, and D. L. Shepelyansky, "Dynamical stochasticity in classical and quantum mechanics", pp. 209-267 in Soviet Scientific Reviews C, vol. 2, Gordon and Breach, New York, 1981.

[David and Barton 1962] F. N. David and D. E. Barton, Combinatorial chance, Griffin, London, 1962.

[Earn and Tremaine 1992] D. J. D. Earn and S. Tremaine, "Exact numerical studies of hamiltonian maps: iterating without roundoff errors", Physica D56 (1992), 1-22.

[Ford 1983] J. Ford, "How random is a coin toss?", Physics Today 36 (1983), 40-47. 
[Gradshteyn and Ryzhik 1980] I. S. Gradsthteyn and I. M. Ryzhik, Table of integrals, series, and products, Academic Press, New York 1980.

[Hammel et al. 1987] S. M. Hammel, J. A. Yorke and C. Grebogi, "Do numerical orbits of chaotic dynamical processes represent true orbits?", J. Complexity $\mathbf{3}$ (1987), 136-145.

[Kaneko 1988] K. Kaneko, "Symplectic cellular automata", Phys. Lett. A129 (1988), 9-16.

[Karney 1983] C. F. F. Karney, "Long time correlations in the stochastic regime", Physica D8 (1983), 360380 .

[Keating 1991] J. P. Keating, "Asymptotic properties of the periodic orbits of the cat maps", Nonlinearity 4 (1991), 277-308.

[Khinchin 1964] A. Ya. Khinchin, Continued fractions, University of Chicago Press, Chicago, 1964.

[Knuth 1981] D. E. Knuth, The art of computer programming, Addison-Wesley, Reading, MA, 1981.

[Marcus 1977] D. A. Marcus, Number Fields, Springer, New York, 1977.
[Morton and Patel 1994] P. Morton and P. Patel, "The Galois theory of periodic points of polynomial maps", Proc. London Math. Soc. 68 (1994), 225-263.

[Percival and Vivaldi 1987] I. C. Percival and F. Vivaldi, "Arithmetical properties of strongly chaotic motions", Physica D25 (1987), 105-130.

[Rannou 1974] F. Rannou, "Numerical studies of discrete plane area-preserving mappings", Astron. Astrophys. 31 (1974), 289-301.

[Ruelle 1978] D. Ruelle, Thermodynamic Formalism: The Mathematical Structures of Classical Equilibrium Statistical Mechanics, Addison-Wesley, Reading, MA, 1978.

[Scovel 1991] C. Scovel, "On symplectic lattice maps", Phys. Lett. A159 (1991), 396-400.

[Vivaldi and Hatjispyros 1992] F. Vivaldi and S. Hatjispyros, "Galois theory of periodic orbits of rational maps", Nonlinearity 5 (1992), 961-978.

[Wang and $\mathrm{Hu}$ 1993] X.-J. Wang and C.-K. Hu, "Anomalous diffusion in dynamical systems: transport coefficients of all order", Phys. Rev. E48 (1993), $728-733$.

Franco Vivaldi, School of Mathematical Sciences, Queen Mary and Westfield College, University of London, Mile End Road, London E1 4NS, England (F.Vivaldi@qmw.ac.uk)

Received October 20, 1994; accepted February 9, 1995 